\title{
Carbon C 11 Erlotinib Hydrochloride
}

National Cancer Institute

\section{Source}

National Cancer Institute. Carbon C 11 Erlotinib Hydrochloride. NCI Thesaurus. Code C103819.

The hydrochloride salt form of the quinazoline derivative erlotinib labeled with the positron-emitting isotope carbon C 11, with potential use in imaging. Competing with adenosine triphosphate, erlotinib reversibly binds to the intracellular catalytic tyrosine kinase domain of the epidermal growth factor receptor (EGFR). Following exposure to this agent, EGFR expression status can be determined and EGFR overexpressing tumor cells can be visualized using positron emission tomography (PET) imaging. This may be useful in determining the tumor cell response to a particular EGFR kinase inhibitor in individual patients. EGFR, a receptor tyrosine kinase, is overexpressed in numerous cancer cell types, and plays a significant role in tumor cell progression. 